\section{If You Hate Vacuum Systems Read On}

\author{
Anthony Buonaquisti \\ Analytical Instrument Facility \\ North Carolina State University \\ Campus Box 7916, Raleigh, NC 24713-7916
}

Let's face it, nobody became a microscopist because they wanted to become an expert in Vacuum Science. The fact is that most of us were shown the proverbial "fly's eye" micrograph and fancied ourselves as the Ansel Adams of Electron Microscopy. As a consequence, during the progress of our careers, we have had to come to grips with Vacuum Science, usually on a "need to know" basis. Specifically, when the system has broken down, we need to know who can fix it ... when they can fix it

and why they can not fix it sooner

Nevertheless, the fact is that we ignore vacuum science at our peril. Although microscopes can withstand a lot of abuse before they catastrophically fail, they respond to poor vacuum practice with poor vacuum quality. In the final analysis it's extremely valuable to have some "vacuum savy".

If the thought of wading through the vacuum texts fills you with dread, don't feel bad. I've taught many classes and most attendees are reluctant students. Over the years I've learned to teach vacuum science with ENTHUSIASM (to keep people interested). I've also learned to begin each class with a quick overview of what happens during the pump-down process of a typical instrument. It seems that although people find vacuum technology a little dry, they find vacuum science quite interesting.

So let's consider the process of pumping down an SEM. We begin with an instrument that contains a lot of gas molecules and we end up with an instrument that contains a lot fewer gas molecules. At first glance this seems pretty boring. Well, the fact is that the process of pump-down is much more interesting than that.

If we were to look inside our vacuum system on a microscopic scale we would see that at atmospheric pressure it contains $10^{19}$ gas molecules $/ \mathrm{cm}^{3}$, the mean distance between molecule - molecule is about $5 \mathrm{~nm}$, the average journey between molecule - molecule collisions (mean free path) is $0.1 \mu \mathrm{m}$ and a freshly cleaved surface would stay clean for about $1 \mathrm{~ns}$ (time for monolayer coverage). The major species would be nitrogen and oxygen with a little water vapor (even in Nevada) and other gases.

When we start pumping we typically use a Rotary (Mechanical) Pump. Rotary pumps work forever provided we maintain them and a modern pump can bring the instruments pressure down to about $10^{-2} \mathrm{Torr}$.

If we were to look inside our vacuum system after this initial pumpdown, we would note that there are $10^{14}$ gas molecules $/ \mathrm{cm}^{3}$, the distance between gas molecules is $0.2 \mu \mathrm{m}$, the mean free path is $1 \mathrm{~cm}$ and the time for monolayer coverage is about $0.1 \mathrm{~ms}$. Finally the major constituents of the vacuum system show a change, with nitrogen and oxygen being less dominant and water vapor being more dominant.

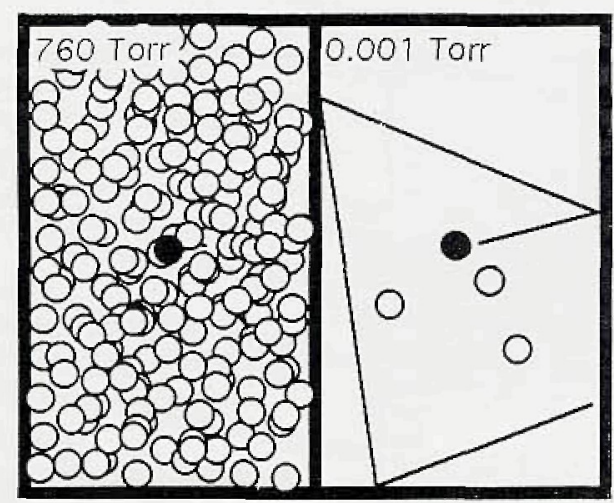

molecule - molecule molecule - wall interactions interactions
At first glance it might seem as if nothing significant has changed. We started off with a lot of molecules and now have a lot less molecules. Isn't that what pumping is all about?

Well, the significant change is that the distance between molecule molecule collisions (the mean free path) is now greater than the diameter of many parts of our instrument. This means that an individual gas molecule in this region is more likely to travel from one inner surface of the instrument to another than it is to collide (or interact) with another gas molecule. As a consequence, each molecule behaves independently and it's behavior depends upon the structure and geometry of the vacuum enclosure. Whereas at higher pressures we could picture the gaseous atmosphere flowing around components during pump-down (viscous flow) now we must picture individual molecules bounding from wall to wall (molecular flow).

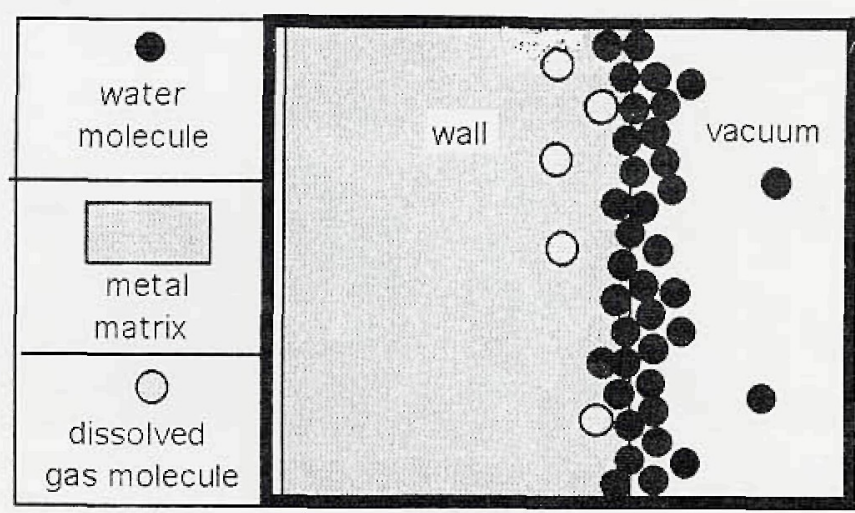

Inner Wall Cross-section

One extremely significant consequence is that an individual molecule is just as likely to head away from the pump as it is to head towards it. Furthermore, an oil molecule within the pump can freely enter the vacuum system unless we specifically design the system to prevent this "backstreaming".

So we begin evacuating our instrument and the consequence of our success is that the way the gas molecules behave changes on us.

If we want to evacuate our system to a greater extent we have to add pumps that operate at lower pressures. These are commonly the Diffusion Pump or the Turbomolecular Pump. Generally they can be relied upon to get a vacuum system down to $10^{-7}$ Torr. This is the high vacuum regime commonly used for SEM's.

If we were to look inside our vacuum system on a microscopic scale we would see that a typical high vacuum instrument contains $10^{9}$ gas molecules I $\mathrm{cm}^{3}$, the mean distance between gas molecules is about $10 \mu \mathrm{m}$, the molecular mean free path is about $10^{5} \mathrm{~cm}$ (over a half mile) and, the time for monolayer (Continued)

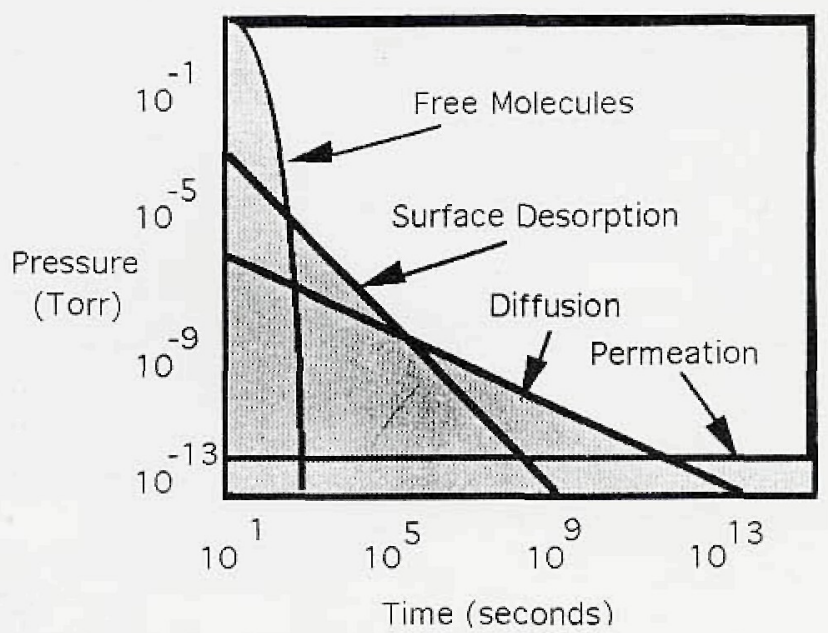

Progress of Evacuation 
coverage is 10 seconds. The major constituent of the free molecule population is water vapor.

Here again, it might seem like little of significance has occurred but once again the vacuum system is significantly different.

At this stage it is significant that water vapor is the major constituent of the free molecules with the system. It's as if our pump only removed the nitrogen and oxygen but ignored the water vapor.

The fact is that the water vapor molecules being pumped under high vacuum conditions come from the walls of the SEM. It turns out that when we evacuate an SEM we don't just remove the atmosphere from inside the instrument, we also remove the molecules that evolve from the walls of the system. The key fact is that the progress of evacuation becomes limited by the rate of water vapor evolution from the walls, rather than the rate at which the pumps can remove water vapor molecules from the free space inside the instrument.

So we continue to evacuate our instrument and the consequence of our success is that the type and source of our gas load changes on us.

Now that we realize that we must consider the molecules that evolve from the inner walls of the instrument we can see that we have to minimize the system's exposure to moisture and other materials that have a vapor pressure. As a consequence it's a good idea to backfill a system with dry nitrogen and also to wear gloves when handling components (and specimens). It's also a good idea to be careful of home built attachments because these might incorporate materials with a high vapor pressure or have machined surfaces that have not been properly cleaned and baked.

If we work on the principle that continued pumping will exhaust even the most abundant reserve of water vapor we might expect our standard SEM to head towards the exalted status of ultra high vacuum.

If we were to look inside an ultra high vacuum system (10-10 Torr) we would note that there are $10^{6}$ gas molecules $/ \mathrm{cm}^{3}$, the distance between gas molecules is $100 \mu \mathrm{m}$, the distance a gas molecule travels between molecule - molecule collisions at $10^{6} \mathrm{~m}$ (greater than 50 miles) and the time for monolayer coverage is about 10,000 seconds. Finally the major constituents of the vacuum system are hydrogen and helium.

At this stage it is significant that the major constituents are hydrogen and helium. These species originate outside our instrument but are so mobile that they can permeate through the very walls of the system.

Nevertheless before we sit back and relax waiting for ultra high vacuum status to reward our patience it might be useful to consider that the process of water vapor evolution is temperature dependant, as is the process of dissolved gas out-diffusion. It turns out that if we just pump even an ideal system it would take a mind numbing $10^{9}$ seconds to reach $10^{-10}$ Tor. Now I like a well pumped vacuum but to wait some 30 years seems a bit excessive.

Ultra high vacuum can be produced and is regularly attained in a whole host of instruments. The trick is to bake the instrument during pump-down. A UHV system such as a Scanning Auger Microprobe, can be pumped from atmosphere to $10^{-10}$ Torr automatically in about $12-24$ hours. Nevertheless, this does mean that these systems have to be designed to withstand the bakeout process and so they tend to be of different design than the standard SEM. Even so, the popularity of cold FEG electron source instruments is bringing UHV into many microscopists lives.

When the science of vacuum is considered, the design and behavior of our instruments make more sense. We also understand why good vacuum practices help us while poor vacuum practices hurt us.

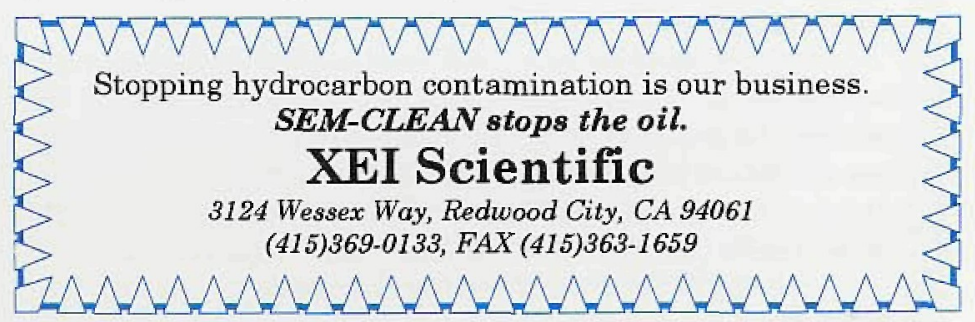

- Accurate quantitative light element analysis

- Fully-focussing (Johansson) optics for optimum resolution

- Minimum detection limits as low as 7ppm

For over 20 years, the name Microspec has been synonymous with quality and reliability. But it is the analytical performance of our X-ray analysis system which sets Microspec apart from the rest. The WDX-3PC spectrometer can easily separate the most difficult peak overlaps, such as S K $\alpha$ and $M o L \alpha$, without the need of spectral deconvolution. And the WDX-3PC can detect light elements down to a few hundred ppm, something other detectors simply cannot achieve. So don't be misled by hype and double-talk. Compare the performance of Microspec against other x-ray systems and you'll see the difference. Call us today and find out how easy it is to meet all of your analytical requirements with the Microspec WDX-3PC.

Microspec Corporation

45950 Hotchkiss Street., Fremont, California 94539

Phone: (510) 656-8820 • Fax: (510) 656-8944

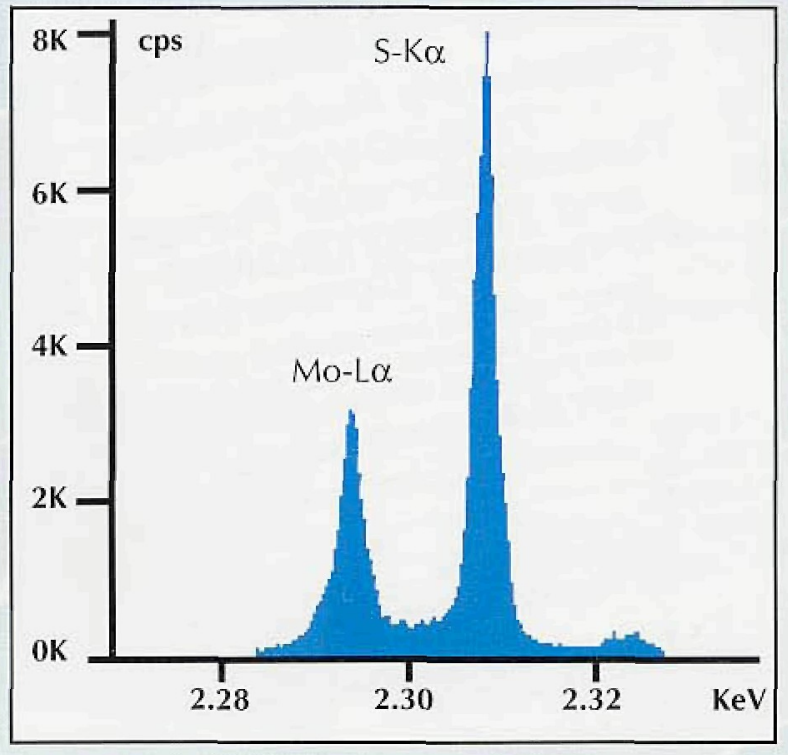

$X$-ray spectrum of $\mathrm{MOS}_{2}$ showing superior resolution of the WDX-3PC. FWHM of $S K \alpha$ is $3 \mathrm{eV}$ ! 